Pacific Journal of Mathematics

ON THE CHARACTERIZATION OF MEASURES OF THE CONE
DUAL TO A GENERALIZED CONVEXITY CONE

Z II ZIEGLER 


\section{ON THE CHARACTERIZATION OF MEASURES OF THE CONE DUAL TO A GENERALIZED CONVEXITY CONE}

\section{ZVI ZiEGLER}

We consider in this paper the cone $C\left(u_{0}, \cdots, u_{n-1}\right)$ of functions which are convex with respect to an Extended Complete Tchebycheffian system $\left\{u_{0}(t), u_{1}(t), \cdots, u_{n-1}(t)\right\}$. The cone dual to $C\left(u_{0}, \cdots, u_{n-1}\right)$ is examined and necessary conditions as well as sufficient conditions for a measure to belong to this cone are developed. The merit of these conditions lies in the fact that they involve only the pattern of sign changes of the measure and related functions, and thus are easily verifiable.

Several applications are given. These include new inequalities for the Euler-Fourier coefficients of functions belonging to given convexity cones. Some new inequalities for the Fourier coefficients of the expansion of a function in a series of orthogonal polynomials are also obtained.

We consider in this paper the cone dual to a generalized convexity cone $C\left(u_{0}, \cdots, u_{n-1}\right)$ with respect to an Extended Complete Tchebycheffian system $\left\{u_{0}(t), u_{1}(t), \cdots, u_{n-1}(t)\right\}$. The substantial role that these cones play in various areas of mathematics, such as moment theory, theory of approximation and interpolation and the theory of differential inequalities is discussed in detail in [5], (see also [4], [11], [6] and [7]). In a recent paper, Cargo [3] obtained independently for the special case when $n=2$ and $u_{0} \equiv 1$, some of the results of [4] and [11].

The dual cone was introduced by S. Karlin and A. Novikoff [4] who found necessary and sufficient conditions for a measure to belong to the dual cone. Applications of the results of [4] to the theory of reliability were later explored by Barlow and Marshall [1]. For the case $n=2$ and $\left(u_{0}(t) \equiv 1, u_{1}(t) \equiv t\right)$ the conditions were stated earlier by Levin and Steckin [8], and a multidimensional version for this special case was recently obtained by Brunk [2].

The necessary and sufficient conditions involve some integral inequalities and thus are not always easily verifiable. Some simple sufficient conditions in terms of equalities and the pattern of sign changes of the measure under examination were also evolved in [4].

In this paper we intend to elaborate on this type of criteria, i.e., necessary conditions as well as sufficient conditions involving only equalities and the pattern of sign changes of the measure. As a byproduct, we obtain the interesting fact that the dual cones are essentially mutually disjoint, e. g. no nontrivial measure can belong both to the dual 
cone of the cone of convex functions and to the cone dual to the cone of monotone functions. Several applications are given in $\S 4$. These include some inequalities for the Euler-Fourier coefficients with respect to the trigonometric system and also for the Fourier coefficients of the expansion of a function in a series of orthogonal polynomials.

We introduce now the generalized convexity cones and their duals. We will not discuss in any detail properties of these cones which can be found elsewhere. The reader is referred to [5] for a thorough discussion of ECT-systems and for the properties of generalized convexity cones which will be used without proof in the sequel.

Let $\left\{u_{i}\right\}_{0}^{n-1}$ be an Extended Complete Tchebycheffian system (ECTsystem) on $[a, b]$. Assume that the functions $u_{i}(t), i=0,1, \cdots, n-1$, admit of the representation

$$
\left\{\begin{array}{l}
u_{0}(t)=w_{0}(t) \\
\vdots \\
\vdots \\
u_{n-1}(t)=w_{c}(t) \int_{a}^{t} w_{1}\left(\xi_{1}\right) \int_{a}^{\xi_{1}} \cdots \int_{a}^{\xi_{n-2}} w_{n-1}\left(\xi_{n-1}\right) d \xi_{n-1} \cdots d \xi_{1}
\end{array}\right.
$$

where $w_{0}(t), \cdots, w_{n-1}(t)$ are continuous strictly positive functions on $[a, b]$. This additional assumption on the set $\left\{u_{i}\right\}_{0}^{n-1}$ entails no loss of generality in the subsequent discussion.

Definition 1. A function $\phi(t)$ defined on $(a, b)$ is said to be convex with respect to the ECT-system $\left\{u_{i}\right\}_{0}^{n-1}$ provided

$$
\left|\begin{array}{ccc}
u_{0}\left(t_{1}\right) & \cdots & u_{0}\left(t_{n+1}\right) \\
\vdots & & \vdots \\
u_{n-1}\left(t_{1}\right) & \cdots & u_{n-1}\left(t_{n+1}\right) \\
\phi\left(t_{1}\right) & \cdots & \phi\left(t_{n+1}\right)
\end{array}\right| \geqq 0, \quad \text { for all } a<t_{1}<\cdots<t_{n+1}<b .
$$

The cone of functions satisfying (2) is referred to as a "generalized convexity cone" and is denoted by $C\left(u_{0}, \cdots, u_{n-1}\right)$.

Throughout the paper, let $d \mu$ denote a signed measure of bounded variation on $(a, b)$ such that for each $\phi(t) \in C\left(u_{0}, \cdots, u_{n-1}\right)$ the integral $\int_{a}^{b} \phi d \mu$ is well defined with infinite values permitted. The dual cone of $C\left(u_{0}, \cdots, u_{n-1}\right)$ is the set of all measures $d \mu$ which satisfy

$$
\int_{a}^{b} \phi(t) d \mu(t) \geqq 0 \text { for all } \phi(t) \in C\left(u_{0}, \cdots, u_{n-1}\right) .
$$

This cone is designated by $C^{*}\left(u_{0}, \cdots, u_{n-1}\right)$.

The integral operators $I_{j}, j=0,1, \cdots, n-1$ are defined by 


$$
\left\{\begin{array}{l}
I_{0} d \mu(t)=-\int_{a}^{t} w_{0}(t) d \mu(t) \\
I_{j} f(t)=-\int_{a}^{t} \mathrm{w}_{j}(t) f(t) d t, \quad j=1,2, \cdots, n-1 .
\end{array}\right.
$$

The following theorem was proved in [4]:

THEOREM A. A signed measure $d \mu$ belongs to the dual cone $C^{*}\left(u_{0}, \cdots, u_{n-1}\right)$ if, and only if

$$
\int_{a}^{b} u_{i}(t) d \mu(t)=0, \quad i=0,1, \cdots, n-1,
$$

and

$$
I_{n-1} I_{n-2} \cdots I_{0} d \mu(t) \geqq 0, \quad \text { for all } a \leqq t \leqq b .
$$

Furthermore, it was shown that the "moment conditions" (5) are equivalent to

$$
I_{j} I_{j-1} \cdots I_{0} d \mu(b)=0, \quad j=0,1, \cdots, n-1 .
$$

The necessary and sufficient conditions stated in Theorem A are in general hard to verify, the main difficulty being the inequalities (6). Therefore, it seems advantageous to seek simpler conditions even if they will not always be both necessary and sufficient. Very weak, but easily verifiable necessary conditions are the "moment conditions" (5). Some simple sufficient conditions which enable us to ascertain that $d \mu \in C^{*}\left(u_{0}, \cdots, u_{n-1}\right)$ by checking its pattern of sign changes were also found. In order to state them we need first introduce some definitions. We adopt the following convention: a signed measure $d \mu$ will be said to have the sign $\varepsilon(\varepsilon$ can be $(+)$ or $(-))$ on a set $s$ if $\varepsilon \mu(s)>0$ and there is no subset $s^{\prime}$ of $s$ for which $\varepsilon \mu\left(s^{\prime}\right)<0$. A function $f(t)$ will be said to have the sign $\varepsilon$ on an interval $I$ if and only if $d \mu=f(t) d t$ has the sign $\varepsilon$ on $I$.

Definition 2. A signed measure $d \mu$ defined on $(a, b)$ is said to possess a first sign there, if there exists an interval extending to the end-point $a$ on which $d \mu$ has a constant sign (this sign will be called the first sign of $d \mu$ ). Similarly, $d \mu$ is said to possess a last sign on $(a, b)$, if there exists an interval extending to the end point $b$ on which $d \mu$ has a constant sign (this sign will be called the last sign of $d \mu$ ).

Definition 3. A signed measure $d \mu$ is said to have $k$ sign changes on $(a, b)$ if there exists a subdivision of $(a, b)$ into disjoint consecutive sets $T_{0}, T_{1}, \cdots, T_{k}$ such that $d \mu$ is of alternating sign on $T_{0}, T_{1}, \cdots, T_{k}$. 
We replaced here the "consecutive intervals" of the corresponding definition employed in [5] by "consecutive sets"-thus allowing a $T_{i}$ to consist of one point only. We note that if the support of the measure consists of a finite number of points or if it is absolutely continuous, the two definitions coincide.

The following theorem stated in [5] (and, in a slightly weaker form, in [4]) is actually true only when one uses the concept of sign changes in the way it is formulated here. The proof involves only minor modifications of the proof presented in [5]. We will not go into details.

THEOREM B. If a nontrivial signed measure dp satisfies the "moment conditions" (5) then it has at least $n$ sign changes. If dre has exactly $n$ sign changes and its last sign is $(+)$, then

$$
d \mu \in C^{*}\left(u_{0}, \cdots, u_{n-1}\right) \text {. }
$$

There exists a wide gap between the necessary "moment conditions" and the strong sufficient conditions stated in Theorem B. The main purpose of this note is to narrow it by obtaining stronger necessary conditions as well as weaker sufficient conditions.

2. Necessary conditions. The first results which we will prove concern the simple cone $C^{*}\left(u_{0}\right)$.

Lemma 1. Let d $\mu$ be a signed measure possessing a first sign and a last sign on $(a, b)$. A necessary condition for $d \mu$ to belong to $C^{*}\left(u_{0}\right)$ is that its first sign be (-) and its last sign be $(+)$.

Proof. Let $d \mu$ be a measure belonging to $C^{*}\left(u_{0}\right)$. Then, by applying Theorem A, we have

$$
\int_{a}^{b} u_{0}(t) d \mu(t)=0
$$

We will first establish that the first sign of $d \mu$ is (-). Indeed, suppose there is an interval $\left(a, t_{1}\right]$ on which $d \mu$ is positive.

Consider the function $\phi(t)$ defined by

$$
\phi(t)=\left\{\begin{array}{ll}
c_{1} u_{0}(t) & a \leqq t \leqq t_{1}, \\
c_{2} u_{0}(t) & t_{1}<t<b,
\end{array} \quad 0<c_{1}<c_{2} .\right.
$$

Clearly, $\phi(t)$ belongs to $C\left(u_{0}\right)$. Compute now 


$$
\begin{aligned}
\int_{a}^{b} \phi(t) d \mu(t)= & c_{1} \int_{a}^{t_{1}} u_{0}(t) d \mu(t)+c_{2} \int_{t_{1}}^{b} u_{0}(t) d \mu(t) \\
& <c_{2} \int_{a}^{b} u_{0}(t) d \mu(t) .
\end{aligned}
$$

Using (8), it follows that

$$
\int_{a}^{b} \phi(t) d \mu(t)<0
$$

which is impossible since $d \mu \in C^{*}\left(u_{0}\right)$.

Similarly, we will now show that the last sign of $d \mu$ is $(+)$. Indeed, assume that there exists an interval $\left[t_{2}, b\right)$ on which $d \mu$ is negative. Consider the function $\psi(t) \in C\left(u_{0}\right)$ defined by

$$
\psi(t)=\left\{\begin{array}{ll}
-c_{2} u_{0}(t) & a<t<t_{2}, \\
-c_{1} u_{0}(t) & t_{2} \leqq t<b,
\end{array} \quad 0<c_{1}<c_{2} .\right.
$$

A computation similar to that performed for $\phi(t)$ yields

$$
\int_{a}^{b} \psi(t) d \mu(t)<0
$$

contrary to the assumption that $d \mu \in C^{*}\left(u_{0}\right)$. This completes the proof of the lemma.

Corollary 1. Let $d \mu$ be a signed measure possessing a finite number of sign changes on $(a, b)$. If d $\mu$ belongs to $C^{*}\left(u_{0}\right)$ then it has an odd number of sign changes and its first sign is (-).

Let now the signed measure $d \mu$ have $2 k-1$ sign changes on $(a, b)$ and let $\left\{T_{i}\right\}_{0}^{2 k-1}$ be the subdivision of $(a, b)$ associated with the sign changes of $d \mu$. Set

$$
S_{i}=T_{2 i-2} \cup T_{2 i-1}, \quad i=1,2, \cdots, k
$$

and let the points $t_{0}, \cdots, t_{2 k}$ be defined by

$$
t_{0}=a, \quad t_{i}=\sup \left\{t: t \in T_{i-1}\right\}, \quad i=1,2, \cdots, 2 k .
$$

Define the numbers $J_{1}, \cdots, J_{k}$ by

$$
J_{i}=\int_{S_{i}} u_{0}(t) d \mu(t), \quad i=1,2, \cdots, k .
$$

The measure $d \mu_{1}$ with the $k$ atomic masses $J_{1}, \cdots, J_{k}$ situated, respectively, at the points $1,2, \cdots, k$ will be referred to in this paper as the measure induced by $d \mu$.

LeMmA 2. Let de have $2 k-1$ sign changes on $(a, b)$ and let its 
first sign be (-). Then $d \mu$ belongs to $C^{*}\left(u_{0}\right)$ on $(a, b)$ if, and only if the measure induced by it belongs to $C^{*}(1)$ on $(0, k+1)$.

Proof. Let $\phi(t)$ be an arbitrary function belonging to $C\left(u_{0}\right)$; then

$$
\begin{aligned}
\int_{a}^{b} \phi(t) d \mu(t) & =\sum_{i=0}^{2 k-1} \int_{T_{i}} \phi(t) d \mu(t) \\
& =\sum_{i=1}^{k}\left[\int_{T_{2 i-i}} \phi(t) d u(t)+\int_{T_{2 i-1}} \phi(t) d \mu(t)\right] \\
& \geqq \sum_{i=1}^{k}\left[\frac{\phi\left(t_{2 i-1}\right)}{u_{0}\left(t_{2 i-1}\right)} \int_{T_{2 i-2}} u_{0}(t) d \mu(t)+\frac{\phi\left(t_{2 i-1}\right)}{u_{0}\left(t_{2 i-1}\right)} \int_{T_{2 i-1}} u_{0}(t) d \mu(t)\right] .
\end{aligned}
$$

The inequality follows from the fact that $\phi(t) / u_{0}(t)$ is non decreasing on $(a, b)$ while $d \mu(t)$ is negative in the first integral and positive in the second.

Using definition (9) we thus obtain

$$
\int_{a}^{b} \phi(t) d \mu(t) \geqq \sum_{i=0}^{k-1} \frac{\phi\left(t_{2 i-1}\right)}{u_{0}\left(t_{2 i-1}\right)} J_{i} .
$$

Suppose now that the induced measure $d \mu$ belongs to $C^{*}(1)$. Then

$$
\sum_{i=1}^{k} a_{i} J_{i} \geqq 0 \text {, for each sequence }\left\{a_{i}\right\}_{1}^{k} \text { belonging to } C(1) \text {. }
$$

Since $\left\{\phi\left(t_{2 i-1}\right) / u_{0}\left(t_{2 i-1}\right)\right\}_{i=1}^{k}$ is a nondecreasing sequence it belongs to $C(1)$. Hence, the right hand side of $(10)$ is nonnegative and $\int_{a}^{b} \phi(t) d \mu(t) \geqq 0$. Since $\phi(t)$ was an arbitrary function of $C\left(u_{0}\right)$, this implies that $\mathrm{d} \mu$ belongs to $C^{*}\left(u_{0}\right)$.

Conversely, suppose that $d \mu \in C^{*}\left(u_{0}\right)$ and let $\left\{\alpha_{i}\right\}_{1}^{k}$ be an arbitrary sequence of $C(1)$. Define the function $\bar{\phi}(t)$ by

$$
\bar{\phi}(t)=a_{i} u_{0}(t), \quad \text { for } t \in S_{i}, i=1,2, \cdots, k,
$$

and note that

$$
\sum_{i=1}^{k} a_{i} J_{i}=\sum_{i=1}^{k} a_{i} \int_{S_{i}} u_{0}(t) d \mu(t)=\int_{a}^{b} \bar{\phi}(t) d \mu(t) \geqq 0 .
$$

The inequality is due to the fact that $\bar{\phi}(t) / u_{0}(t)$ is a nondecreasing function, i.e., that $\bar{\phi}(t)$ belongs to $C\left(u_{0}\right)$.

Since the sequence $\left\{a_{i}\right\}_{1}^{k}$ was an arbitrary sequence of $C(1)$, this completes the proof of the lemma.

Appealing to Corollary 1 , we can deduce

Corollary 2. Let du be a measure of $C^{*}\left(u_{0}\right)$ possessing a finite 
number of sign changes on $(a, b)$. Then, either the induced measure $d \mu_{1}$ is the trivial measure or it has an odd number of sign changes and its first sign is (-).

Observe next that if the induced measure $d \mu_{1}$ has an odd number of sign changes, the discussion preceding Lemma 2 can be applied to $d \mu_{1}$ and a measure $d \mu_{2}$, induced by $d \mu_{1}$, can be obtained. To this end, we only have to substitute $u_{0}(t) \equiv 1$ in $(9)$ and replace $(a, b)$ by $(0, k+1)$. By Corollary $2, d \mu_{2}$ is either trivial or it has an odd number of sign changes. Thus, if $d \mu_{2}$ is nontrivial, we can define a measure $d \mu_{3}$ induced by $d \mu_{2}$. This process can be continued as long as the induced measure is nontrivial.

Lemma 3. Let $d \mu$ be a measure of $C^{*}\left(u_{0}\right)$ possessing a finite number of sign changes on $(a, b)$. Then the sequence of nontrivial successively induced measures $d \mu_{1}, d \mu_{2}, \cdots$, is finite.

Proof. Observe that the induced measures $d \mu_{1}, d u_{2}, \cdots$, have finite supports. Note next that the number of points in the support of $d \mu_{i+1}, i=1,2, \cdots$, is at most half the number of points in the support of $d \mu_{i}, i=1,2, \cdots$. Hence, the assertion of the lemma follows.

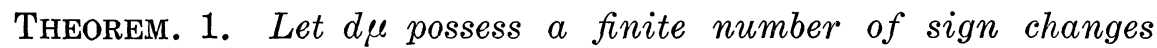
on $(a, b)$. Necessary and sufficient conditions for de to belong to $C^{*}\left(u_{0}\right)$ are: (a) that it satisfy (8), and (b) that de and each measure in the finite sequence of nontrivial successively induced measures $d \mu_{1}, d \mu_{2}, \cdots$, exhibit the pattern of sign changes specified in Lemma 1 .

Proof. Necessity. The necessity of (a) follows from Theorem A. The necessity of (b) follows by a repeated application of Corollary 2 .

Sufflciency. Let $d \mu_{N}$ be the last nontrivial measure in the sequence, so that $d \mu_{N+1}$ is the trivial measure.

Since $d \mu$ has a finite number of sign changes, each nontrivial measure $d \mu_{i}, i=1, \cdots, N$, also has a finite number of sign changes. Since, by assumption, the measures exhibit the pattern of sign changes specified in Lemma 1, they satisfy the requirements of Lemma 2.

By Lemma 2 , if $d \mu_{i+1}, i=1, \cdots, N$ belongs to $C^{*}(1)$, then so does $d \mu_{i}$. Furthermore, if $d \mu_{1}$ belongs to $C^{*}(1)$ then $d \mu$ belongs to $C^{*}\left(u_{0}\right)$. Thus, the fact that $d \mu_{N+1}$, the trivial measure, belongs to $C^{*}(1)$, implies that $d \mu$ belongs to $C^{*}\left(u_{0}\right)$ and the theorem is proved.

We next derive necessary conditions for a measure possessing a first sign and a last sign on $(a, b)$ to belong to $C^{*}\left(u_{0}, \cdots, u_{n-1}\right)$. 


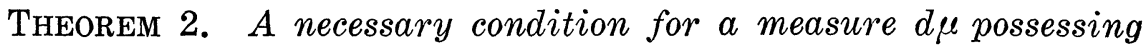
a first sign and $a$ last sign on $(a, b)$ to belong to $C^{*}\left(u_{0}, \cdots, u_{n-1}\right)$ is that its first sign be $(-1)^{n}$ and its last sign be $(+)$.

Proof. The proof proceeds by induction on $n$. For $n=1$, the assertion is simply a restating of Lemma 1 . Assuming that the assertion is valid for $n \leqq k-1$, we will now prove it for $n=k$.

We introduce the first order differential operators (see [5])

$$
D_{j} f(t)=\frac{d}{d t}\left[\frac{1}{w_{j}(t)} f(t)\right], \quad j=0,1, \cdots, n-1,
$$

where the $w_{i}^{\prime} s$ are the functions introduced in (1).

Let now $d \mu$ be a measure of $C^{*}\left(u_{0}, \cdots, u_{k-1}\right)$ possessing a first sign and a last sign on $(a, b)$. Using integration by parts and the definitions (4) and (12), we find

$$
\begin{aligned}
\int_{a}^{b} \phi(t) d \mu(t) & =\int_{a}^{b} \frac{\phi(t)}{w_{0}(t)} w_{0}(t) d \mu(t) \\
& =-\left.\frac{\phi(t)}{w_{0}(t)} I_{0} d \mu(t)\right|_{a} ^{b}+\int_{a}^{b}\left[D_{0} \phi(t)\right]\left[I_{0} d \mu(t)\right] d t .
\end{aligned}
$$

The integrated part vanishes, since $I_{0} d \mu(b)=0$ is a necessary condition by Theorem A. It is very easy to see (cf. [11] or [5]) that the set of functions $\left\{D_{0} \phi(t) \mid \phi(t) \in C\left(u_{0}, \cdots, u_{k-1}\right)\right\}$ comprises a generalized convexity cone. This cone is called the first "reduced" cone, and is denoted, in terms of its basic ECT-system, by $C\left(D_{0} u_{1}, \cdots, D_{0} u_{k-1}\right)$. Thus, (13) implies that a necessary condition for $d \mu$ to belong to $C^{*}\left(u_{0}, \cdots, u_{k-1}\right)$ is that $I_{0} d \mu(t) d t$ belong to $C^{*}\left(D_{0} u_{1}, \cdots, D_{0} u_{k-1}\right)$.

Since $d \mu$ has a first sign and a last sign, so does $I_{0} d \mu(t) d t$. Utilizing now the fact that the condition on the pattern of signs formulated in the theorem depends only on the order of the cone, i.e. on the number of functions in its basic ECT-system, we can apply the induction hypothesis. We thus deduce that the first sign of $I_{0} d \mu(t)$ is $(-1)^{k-1}$ and its last sign in $(+)$.

Note further that

$$
I_{0} d \mu(t)=-\int_{a}^{t} u_{0}(t) d \mu(t),
$$

and that, using relation (8), which is valid by Theorem A, we also have

$$
I_{0} d \mu(t)=\int_{t}^{b} u_{0}(t) d \mu(t) .
$$

Relations (14) and (15) imply that the first sign of $d \mu(t)$ is opposite 
to that of $I_{0} d \mu(t)$ and that the last sign of $d \mu(t)$ is the same as that of $I_{0} d \mu(t)$. This completes the induction step and thereby the theorem is proved.

The set of measures of a dual cone $C^{*}\left(u_{0}, \cdots, u_{n-1}\right)$ which possess a first sign on $(a, b)$ is a subcone. This subcone will be called the restricted dual cone. Note that the trivial measure does not belong to the restricted dual cone.

The condition on the pattern of signs proved in Theorem 2 readily yield

CoROLlaRy 3. A restricted dual cone of odd order and a restricted dual cone of even order are always mutually disjoint.

Note that in Corollary 3, the cones may be based on different ECT-systems. For a fixed ECT-system, a more comprehensive result in this direction is true, viz.

THEOREM 3. Let an ECT-system be given. Two dual cones with respect to this system which are of different orders have only the trivial measure in common.

Proof. Consider $C^{*}\left(u_{0}, \cdots, u_{n-1}\right)$ and $C^{*}\left(u_{0}, \cdots, u_{k-1}\right)$ with $n>k$. Let $d \mu$ be a measure belonging to $C^{*}\left(u_{0}, \cdots, u_{k-1}\right)$. Then the necessary conditions of Theorem A imply that

$$
I_{k-1} I_{k-2} \cdots I_{0} d \mu(t) \geqq 0, \quad \text { for } a \leqq t \leqq b .
$$

Suppose now that $d \mu$ belongs also to $C^{*}\left(u_{0}, \cdots, u_{n-1}\right)$. By repeated integration by parts similar to that performed in (13), we find

$$
\begin{aligned}
\int_{a}^{b} \phi(t) d \mu(t) & =-\left.\frac{\phi(t)}{w_{0}(t)} I_{0} d \mu(t)\right|_{a} ^{b}-\left.\sum_{j=1}^{k-1} \frac{D_{j-1} \cdots D_{0} \phi(t)}{w_{j}(t)} I_{j} \cdots I_{0} d \mu(t)\right|_{a} ^{b} \\
& +\int_{a}^{b}\left[D_{k-1} D_{k-2} \cdots D_{0} \phi(t)\right]\left[I_{k-1} I_{k-2} \cdots I_{0} d \mu(t)\right] d t .
\end{aligned}
$$

Tne integrated part vanishes by virtue of the conditions (7) which are necessary conditions for $d \mu$ to belong to $C^{*}\left(u_{0}, \cdots, u_{n-1}\right)$. Hence, as in the proof of Theorem 2 , we deduce that a necessary condition for $d \mu$ to belong to $C^{*}\left(u_{0}, \cdots, u_{n-1}\right)$ is that $I_{k-1} I_{k-2} \cdots I_{0} d \mu(t) d t$ belong to the dual to the $k$-th "reduced" cone

$$
C^{*}\left(D_{k-1} \cdots D_{0} u_{k}, D_{k-1} \cdots D_{0} u_{k+1}, \cdots, D_{k-1} \cdots D_{0} u_{n-1}\right) .
$$

This is a dual cone of order $n$ - $k$, so that by Theorem $\mathrm{B}$, a necessary condition for this to happen, is that either $I_{k-1} \cdots I_{0} d \mu(t)$ have at least $n-k$ sign changes on $(a, b)$, or that $I_{k-1} \cdots I_{0} d \mu(t) \equiv 0$. Since 
(16) has to be satisfied, we deduce that $I_{k-1} \cdots I_{0} d \mu(t) \equiv 0$; this is equivalent to $d \mu$ being the trivial measure, so that the proof is complete.

We have seen that for a fixed ECT-system, the intersection of two dual cones of different order contains only the trivial measure. The question of the structure and properties of unions of such cones will be explored by the author in a future publication.

3. Sufficient conditions. We have, in the last section, strengthened the necessary conditions given by Theorem $\mathrm{A}$, by adding that if a signed measure $d \mu$ belongs to $C^{*}\left(u_{0}, \cdots, u_{n-1}\right)$ and possesses a first sign and a last sign, then its first sign must be $(-1)^{n}$ and its last sign must be $(+)$.

We shall obtain in this section weaker sufficient conditions than those specified in Theorem $\mathrm{B}$.

Let the functions $U_{i}(\mu ; t), i=0,1, \cdots, n-1$, be defined by

$$
U_{i}(\mu ; t)=\int_{a}^{t} u_{i}(t) d \mu(t), \quad i=0,1, \cdots, n-1 .
$$

These functions are smoother than the measure $d \mu(t)$ and therefore it is sometimes easier to check their respective patterns of signs than to check the pattern of signs of $d \mu$.

THEOREM 4. Let de satisfy the "moment conditions" (5) and let its first sign be $(-1)^{m}$ and its last sign be $(+)$. If there exists a $j$, $0 \leqq j \leqq n-1$, such that $U_{j}(\mu ; t)$ has at most $n-1$ sign changes on $(a, b)$, then $d \mu \in C^{*}\left(u_{0}, \cdots, u_{n-1}\right)$.

Proof. The proof proceeds by induction. Let $\left(u_{0}, \cdots, u_{m-1}\right), m \geqq 1$, be an arbitrary ECT-system. (Note that this is a completely arbitrary ECT-system. We have chosen to denote its functions by $\left(u_{0}, \cdots, u_{m-1}\right)$ in order to be able to avail ourselves of other theorems of the paper without undue change of notation).

Assume that $d \mu(t)$ satisfies the "moment conditions" (5) (where $n$ is replaced by $m$ ), and that its first sign is $(-1)^{m}$ and its last sign is $(+)$. Assume further that $U_{0}(\mu ; t)$ has at most $m-1$ sign changes on $(a, b)$. We will now show that these assumptions imply that

$$
d \mu \in C^{*}\left(u_{0}, \cdots, u_{m-1}\right) .
$$

We note that $U_{0}(\mu ; t)=-I_{0} d \mu(t)$, and observe that (13) and (5) imply that

$$
\int_{a}^{b} \phi(t) d \mu(t)=\int_{a}^{b}\left[D_{0} \phi(t)\right]\left[I_{0} d \mu(t)\right] d t
$$


Thus, it will suffice if we show that $I_{0} d \mu(t) d t$ belongs to

$$
C^{*}\left(D_{0} u_{1}, \cdots, D_{0} u_{m-1}\right) \text {. }
$$

Relations (18) and (5) imply that $I_{0} d \mu(t) d t$ satisfies the $m-1$ "moment conditions" with respect to $\left(D_{0} u_{1}, \cdots, D_{0} u_{m-1}\right)$. Hence, by Theorem $\mathrm{B}$, it has at least $m-1$ sign changes. However, by our assumption, $I_{0} d \mu(t)$ has at most $m-1$ sign changes, so that it must have exactly $m-1$ sign changes. Furthermore, following the same reasoning as in the proof of Theorem 2, we deduce that the first sign of $I_{0} d \mu(t)$ is $(-1)^{m-1}$ and its last sign is $(+)$. Therefore, by Theorem $B$, $I_{0} d \mu(t) d t$ belongs to $C^{*}\left(D_{0} u_{1}, \cdots, D_{0} u_{m-1}\right)$.

We have thus proved that if an ECT-system of order $m, m \geqq 1$, is given and $d \mu$ is a signed measure with first $\operatorname{sign}(-1)^{m}$ and last sign $(+)$ satisfying the corresponding "moment conditions", then the condition that $U_{0}(\mu ; \mathrm{t})$ have at most $m-1$ sign changes on $(a, b)$ implies that $d \mu$ belongs to the corresponding dual cone.

Assume now that we have established that, given any ECT-system of order $m$ and a signed measure $d \mu$ satisfying the corresponding "moment conditions" and having the appropriate first and last signs, the condition that $U_{r-1}(\mu ; t), 1 \leqq r<m$, have at most $m-1$ sign changes on $(a, b)$ implies that $d \mu$ belongs to the corresponding dual cone.

We wish to show that the same conclusion is implied by the condition that $U_{r}(\mu ; t)$ have at most $m-1$ sign changes. This will be the induction step and thereby the validity of the theorem will be established.

Let $d \mu(t)$ be a signed measure whose first sign is $(-1)^{m}$ and whose last sign is $(+)$ and let it satisfy (5). Furthermore, assume that $U_{r}(\mu ; t)$ has at most $m-1$ sign changes. We wish to show that these assumptions together with the induction hypothesis imply that $d \mu \in C^{*}\left(u_{0}, \cdots, u_{m-1}\right)$. It will suffice, as explained earlier, if we show that $I_{0} d \mu(\mathrm{t}) d t \in C^{*}\left(D_{0} u_{1}, \cdots, D_{0} u_{m-1}\right)$.

Consider the ECT-system $\left(D_{0} u_{1}, \cdots, D_{0} u_{m-1}\right)$ and define

$$
U_{i}^{*}(\mu ; t)=\int_{a}^{t} D_{0} u_{i+1}(t) d \mu(t), \quad i=0,1, \cdots, m-2 .
$$

In the case where $d \mu(t)=f(t) d t$, the left hand side of (19) will be written as $U_{i}^{*}(f ; t)$.

Integration by parts similar to that performed in (13) yields

$$
U_{j-1}^{*}\left(I_{0} d \mu ; t\right)=\frac{u_{j}(t)}{w_{0}(t)} I_{0} d \mu(t)+U_{j}(\mu ; t), \quad j=1,2, \cdots, m-1 .
$$

Note that the functions $U_{j}^{*}(\mu ; t), j=0,1, \cdots, m-2$ are defined with respect to the ECT-system of order $m-1\left(D_{0} u_{1}, \cdots, D_{0} u_{m-1}\right)$ in 
exactly the same way that $U_{j}(\mu ; t), j=0,1, \cdots, m-1$ were defined in (17) with respect to $\left(u_{0}, \cdots, u_{m-1}\right)$. Note further that our assumptions on $d \mu$ imply that the first sign of $I_{0} d \mu(t)$ is $(-1)^{m-1}$ and its last sign is $(+)$ and that $I_{0} d \mu(t)$ satisfies the "moment conditions" with respect to $\left(D_{0} u_{1}, \cdots, D_{0} u_{m-1}\right)$. Thus, if we show that $U_{r-1}^{*}\left(I_{0} d \mu ; t\right)$ has at most $m-2$ sign changes, the induction hypothesis, which is applicable since $r-1<m-1$, will imply that

$$
I_{0} d \mu(t) \in C^{*}\left(D_{0} u_{1}, \cdots, D_{0} u_{m-1}\right) \text {. }
$$

We start with an analysis of the patterns of signs of $U_{r-1}^{*}\left(I_{0} d \mu ; t\right)$ and $U_{r}(\mu ; t)$. Since the first sign of $I_{0} d \mu(t)$ is $(-1)^{m-1}$ the same is true for $U_{r-1}^{*}\left(I_{0} d \mu ; t\right)$. Similarly, since the first sign of $d \mu$ is $(-1)^{m}$ the same is true for $U_{r}(\mu ; t)$. On the other hand, the last signs of both $d \mu$ and $I_{0} d \mu(t)$ are $(+)$ so that the last signs of both $U_{r-1}^{*}\left(I_{0} d \mu ; t\right)$ and $U_{r}(\mu ; t)$ are $(-)$.

Let $\nu$ be the number of sign changes of $U_{r-1}^{*}\left(I_{0} d \mu ; t\right)$; the above analysis of first and last signs implies that

$$
\nu \equiv m(\bmod 2) \text {. }
$$

Suppose now that $U_{r-1}^{*}\left(I_{0} d \mu ; t\right)$ has more than $m-2$ sign changes. Then, by (21), it must have at least $m$ sign changes. We assert that this is incompatible with the assumption that $U_{r}(\mu ; t)$ has at most $m-1$ sign changes.

We divide the proof of this assertion in two parts.

(a) Let $\left(T_{0}^{*}, \cdots, T_{\nu}^{*}\right)$ be the subdivision of $(a, b)$ associated with the sign changes of $U_{r-1}^{*}\left(I_{0} d \mu ; t\right)$ and let $\left\{t_{i}^{*}\right\}_{1}^{\nu}$, the points of sign change of $U_{r-1}^{*}\left(I_{0} d \mu ; t\right)$, be defined by $t_{i}^{*}=\sup \left\{t: t \in T_{i-1}^{*}\right\}, i=1,2, \cdots, \nu$. Then $U_{r}(\mu ; t)$ changes sign at least once in $\left(a, t_{1}^{*}\right)$.

Note first that $U_{r-1}^{*}\left(I_{0} d \mu ; t\right)$ is a continuous function, so that the points $t_{i}^{*}, i=1,2, \cdots, \nu$, are among its zeros. By considering the pattern of signs of $U_{r-1}^{*}\left(I_{0} d \mu ; t\right)$ we see that $(-1)^{m-1} U_{r-1}^{*}\left(I_{0} d \mu ; t\right)$ is positive on $\left(a, t_{1}^{*}\right]$ and changes its sign to negative at $t_{1}^{*}$. Hence, there must exist a point $x, a<x<t_{1}^{*}$, such that

$$
(-1)^{m-1} D_{0} u_{r-1}(x) I_{0} d \mu(x)<0 \text {. }
$$

Moreover, since $D_{0} u_{r-1}(t)$ is strictly positive on $(a, b)$, we have

$$
(-1)^{m-1} I_{0} d \mu(x)<0 \text {. }
$$

This inequality, taken together with relation $(20)$ and the fact that $u_{j}(t)$ and $w_{0}(t)$ are strictly positive on $(a, b)$, implies that

$$
(-1)^{m-1} U_{r}(\mu ; x)>0 \text {. }
$$

However, we know that the first sign of $(-1)^{m} U_{r}(\mu ; t)$ is $(+)$. 
Hence, a sign change must have occurred for some $\bar{t}, a<\bar{t}<x<t_{1}^{*}$. This completes the first part.

(b) In each interval $\left[t_{i}^{*}, t_{i+1}^{*}\right), i=1,2, \cdots, \nu$ (where $t_{\nu+1}^{*}=b$ ), the function $U_{r}(\mu ; t)$ has at least one point of sign change.

Indeed, with no loss of generality we may assume that $U_{r-1}^{*}\left(I_{0} d \mu ; t\right)$ is positive for $t \in\left[t_{i}^{*}, t_{i+1}^{*}\right]$. Since there exists a point $s, t_{i}^{*}<s<t_{i+1}^{*}$ such that $U_{r-1}^{*}\left(I_{0} d \mu ; s\right)>0$ and we also have $U_{r-1}^{*}\left(I_{0} d \mu ; t_{i+1}^{*}\right)=0$, it follows that there exists a point $x_{2}, t_{i}^{*}<x_{2}<t_{i+1}^{*}$ for which

$$
D_{0} u_{r-1}\left(x_{2}\right) I_{0} d \mu\left(x_{2}\right)<0 \text {. }
$$

Since $U_{r-1}^{*}\left(I_{0} d \mu ; x_{2}\right) \geqq 0$, relation (20) implies that $U_{r}\left(\mu ; x_{2}\right)>0$.

On the other hand, $t_{i}^{*}$ is a point where $U_{r-1}^{*}\left(I_{0} d \mu ; t\right)$ changes sign from negative to positive. Hence, for each $y, y<t_{i}^{*}$, there exists a point $x_{1}, y<x_{1}<t_{i}^{*}$ such that $D_{0} u_{r-1}\left(x_{1}\right) I_{0} d \mu\left(x_{1}\right)>0$ and

$$
U_{r-1}^{*}\left(I_{0} d \mu ; x_{1}\right) \leqq 0 .
$$

We deduce from $(20)$ that $U_{r}\left(\mu ; x_{1}\right)<0$. Hence, $U_{r}(\mu ; t)$ must change sign between $x_{1}$ and $x_{2}$. Noting that $y$ was an arbitrary point satisfying $y<t_{i}^{*}$, we conclude that there exists a point $x, t_{i}^{*} \leqq x<x_{2}<t_{i+1}^{*}$, which is a point of sign change for $U_{r}(\mu ; t)$.

Combining parts (a) and (b) we see that $U_{r}(\mu ; t)$ has at least as many sign changes as $U_{r-1}^{*}\left(I_{0} d \mu ; t\right)$. Thus, if $U_{r-1}^{*}\left(I_{0} d \mu ; t\right)$ has at least $m$ sign changes, then so does $U_{r}(\mu ; t)$, proving the assertion. This completes the proof of Theorem 4 .

Remark. The conditions specified in Theorem 4 are weaker than those specified in Theorem B. Indeed, if $d \mu$ has exactly $n$ sign changes on $(a, b)$ and conditions (5) are satisfied, it follows easily that the functions $U_{i}(\mu ; t), i=0,1, \cdots, n-1$ can have at most $n-1$ sign changes. The converse is not true. There exist, in fact, examples such that $d \mu$ possesses in excess of $n$ sign changes, while there exists a $j, 0 \leqq j \leqq n-1$, such that $U_{j}(\mu ; t)$ has no more than $n-1$ sign changes.

4. Applications. In this section we discuss several applications of the foregoing analysis to Fourier series [part a)] and to expansions of functions in terms of orthogonal polynomials [part b)]. Some of the results stated here might have been discussed elsewhere, but even in that case, the power of our criteria is exemplified by the simplicity of the derivation of the results. Thanks are due to Prof. B. Schwarz who drew our attention to the fact that a special case of assertion (B) below is discussed in [9, Vol. 2, p. 81]. This is the only case which, to the best of our knowledge, has been discussed in the literature. 
The inequalities discussed in this section are necessary conditions for functions to be included in given convexity cones. The following converse problem is suggested:

Determine a set of conditions on the Fourier coefficients of a function which will be sufficient to insure the inclusion of the function in a given convexity cone.

(a) Fourier series. Let $f(t)$ denote throughout this subsection a function of $L_{2}(-\pi, \pi)$ and let

$$
\frac{a_{0}}{2}+\sum_{k=1}^{\infty} a_{k} \cos k t+b_{k} \sin k t
$$

be the corresponding Fourier series.

We shall present the inequalities for the Euler-Fourier coefficients of functions belonging to convexity cones in the form of a series of assertions.

(A) Let $f(t)$ be monotone nondecreasing on $(-\pi, \pi)$. Then

$$
(-1)^{n+1} b_{n} \geqq 0, \quad n=1,2, \cdots .
$$

Proof. The assertion is equivalent to the relation

$$
(-1)^{n+1} \int_{-\pi}^{\pi} f(t) \sin n t d t \geqq 0, \quad \text { for each } f(t) \text { of } C(1) .
$$

Thus, we have to show that $d \mu_{A}(t)=(-1)^{n-1} \sin n t d t$ belongs to $C^{*}(1)$. We note first that the last sign of $d \mu_{A}$ is $(+)$ and that $d \mu_{A}$ is odd. Hence, it has the pattern of signs specified in Lemma 2. The zeros of $d \mu_{A}$ inside $(-\pi, \pi)$, which are simple zeros and therefore points of sign change for $d \mu_{A}$, are the points $\{-\pi+k \pi / n, k=1,2, \cdots, 2 n-1\}$. Thus, we have

$$
J_{i}=\int_{-\pi+(2 i \pi / n)}^{-\pi+\{(2 i+2) / n\} \pi}(-1)^{n+1} \sin n t d t, \quad i=0,1, \cdots, n-1,
$$

and this expression is zero for each $i, 0 \leqq i \leqq n-1$. Hence, the measure induced by $d \mu_{A}$ belongs to $C^{*}(1)$, and by Lemma 2 so does $d \mu_{A}$.

(B) Let $f(t)$ be convex on $(-\pi, \pi)$. Then

$$
(-1)^{n} a_{n} \geqq 0, \quad n=1,2, \cdots .
$$

Proof. The assertion is equivalent to the relation

$$
(-1)^{n} \int_{-\pi}^{\pi} f(t) \cos n t d t \geqq 0 \quad \text { for all } f(t) \text { of } C(1, t) \text {. }
$$


Thus, we have to show that $d \mu_{B}(t)=(-1)^{n} \cos n t d t$ belongs to $C^{*}(1, t)$. Observe that

$$
I_{0} d \mu_{B}(t)=-\int_{-\pi}^{t}(-1)^{n} \cos n x d x=\frac{(-1)^{n+1} \sin n t}{n},
$$

so that $I_{0} d \mu_{B}(\pi)=0$ and $I_{0} d \mu_{B}(t) d t \in C^{*}(1)$. By the remark following equation (18), these are sufficient conditions for $d \mu_{B}(t)$ to belong to $C^{*}(1, t)$.

(C) Let $f(t)$ be monotone nondecreasing on $(-\pi, \pi)$. Then

$$
\left|\frac{b_{k n}}{n}\right| \leqq\left|b_{k}\right|, \quad \begin{aligned}
& k=1,2, \cdots, \\
& n=1,2, \cdots .
\end{aligned}
$$

Proof. In view of (23), we have to show that

$$
(-1)^{k+1} b_{k} \geqq(-1)^{k n+1} b_{k n} / n,
$$

i.e., that $d \mu_{C}(t)=\left[(-1)^{k+1} \sin k t-(-1)^{k n+1}(\sin k n t / n)\right] d t$ belongs to $C^{*}(1)$. We note that

$$
I_{0} d \mu_{C}(\pi)=\int_{-\pi}^{\pi} d \mu_{C}(\pi)=0 .
$$

From the well known inequality (see e.g. [9])

$$
|\sin N x| \leqq N|\sin x|, \quad N=1,2, \cdots,
$$

it follows that

$$
\left|\frac{\sin k n t}{n}\right| \leqq|\sin k t|, \quad \begin{aligned}
& k=1,2, \cdots, \\
& n=1,2, \cdots,
\end{aligned}
$$

so that the sign of $d \mu_{C}(t)$ is identical, for each $t$, with the sign of $(-1)^{k+1} \sin k t$. Thus, the first sign of $d \mu_{C}$ is $(-)$ and its last sign is $(+)$, so that $d \mu_{C}$ has the pattern of sign changes specified in Lemma 2. Noting that the points of sign change of $d \mu_{C}$ inside $(-\pi, \pi)$ are $\{-\pi+(i \pi / k), i=1, \cdots, 2 k-1\}$, we have

$$
\begin{array}{r}
J_{i}=\int_{-\pi+(2 i \pi / k)}^{-\pi+\{(2 i+2) \pi / k\}}\left[(-1)^{k+1} \sin k t-(-1)^{k n+1} \frac{\sin k n t}{n}\right] d t, \\
i=0,1, \cdots, k-1 .
\end{array}
$$

This expression is zero for each $i, i=0,1, \cdots, k-1$. Thus, by Lemma 2 , $d \mu_{C}$ belongs to $C^{*}(1)$.

(D) Let $f(t)$ be conver on $(-\pi, \pi)$. Then 


$$
\left|a_{k}\right| \geqq\left|a_{n k}\right|, \quad k=1,2, \cdots,
$$

Proof. In view of (24), we have to show that

$$
(-1)^{k} a_{k} \geqq(-1)^{n k} a_{n k},
$$

i.e., that $d \mu_{D}(t)=\left[(-1)^{k} \cos k t-(-1)^{n k} \cos n k t\right] d t$ belongs to $C^{*}(1, t)$. We note that

$$
I_{0} d \mu_{D}(t)=-\int_{-\pi}^{t} d \mu_{D}(t)=\frac{1}{k}\left[(-1)^{k+1} \sin k t-(-1)^{k n+1} \frac{\sin k n t}{n}\right] .
$$

Thus, $I_{0} d \mu_{D}(\pi)=0$ and, by assertion $\left.C\right), I_{0} d \mu_{D}(t)$ belongs to $C^{*}(1)$. These conditions imply that $d \mu_{D}(t)$ belongs to $C^{*}(1, t)$.

(E) Let $f(t)$ be monotone nondecreasing on $(-\pi, \pi)$. Then

$$
\sum_{k=1}^{n} b_{k}+\frac{1}{2} b_{n+1} \geqq 0, \quad n=1,2, \cdots .
$$

Proof. We need only observe that

$$
d \mu_{E}(t)=\left[\sum_{k=1}^{n} \sin k t+\frac{1}{2} \sin (n+1) t\right] d t
$$

is nonnegative for $0 \leqq t \leqq \pi$ (see [9]) and odd. The "moment condition" $I_{0} d \mu_{E}(\pi)=0$ is clearly satisfied, and the previous observation implies that there exists precisely one sign change. The assertion follows then by appealing to Theorem B. Note that if $n$ is odd, relations (28) and (23) imply

$$
\sum_{k=1}^{n} b_{k} \geqq 0, \quad \text { for each odd } n .
$$

(F) Let $f(t)$ be convex on $(-\pi, \pi)$. Then

$$
\sum_{k=1}^{n} k a_{k}+\frac{(n+1)}{2} a_{n+1} \leqq 0, \quad n=1,2, \cdots .
$$

Proof. Set $d \mu_{F}(t)=-\left[\sum_{k=1}^{n} k \cos k t+\{(n+1) / 2\} \cos (n+1) t\right] d t$; it is easily seen that $I_{0} d \mu_{F}(\pi)=0$ and that $I_{0} d \mu_{F}(t)=d \mu_{E}(t)$ belongs to $C^{*}(1)$. These conditions imply that $d \mu_{F}$ belongs to $C^{*}(1, t)$. Note also that relations (30) and (24) imply that

$$
\sum_{k=1}^{n} k a_{k} \leqq 0, \quad \text { for each odd } n .
$$


(G) Let $f(t)$ be monotone nondecreasing on $(-\pi, \pi)$. Then

$$
\sum_{k=1}^{n} k(n+1-k) b_{k} \geqq 0, \quad \text { for each odd } n .
$$

Proof. Set $d \mu_{G}(t)=\left[\sum_{k=1}^{n} k(n+1-k) \sin k t\right] d t$. Straight computation yields

$$
I_{0} d \mu_{G}(t)=\sum_{k=1}^{n}(n+1-k) \cos k t+C .
$$

We recall the equation (see [9])

$$
\sum_{k=1}^{n}(n+1-k) \cos k t+\frac{n+1}{2}=\frac{1}{2}\left[\frac{\sin (n+1) t / 2}{\sin t / 2}\right]^{2} .
$$

The right hand side of (33) differs from $I_{0} d \mu_{G}(t)$ by a constant at most. However, for an odd $n$ the right hand side of (33) vanishes for $t=\pi$ and so does $I_{0} d \mu_{G}(t)$ as is clear from the definition of $d \mu_{G}$. Therefore we have

$$
I_{0} d \mu_{G}(t)=\frac{1}{2}\left[\frac{\sin (n+1) t / 2}{\sin t / 2}\right]^{2}
$$

so that $I_{0} d \mu_{G}(t)$ is nonnegative on $(-\pi, \pi)$ and vanishes for $t=\pi$. This implies, using Theorem $\mathrm{A}$, that $d \mu_{G}$ belongs to $C^{*}(1)$.

(H) Let $f(t)$ be convex on $(-\pi, \pi)$. Then

$$
\sum_{k=1}^{n} k^{2}(n+1-k) a_{k} \leqq 0, \quad \text { for each odd } n .
$$

Proof. This assertion follows from assertion (G) in precisely the same way as $(\mathrm{F})$ followed from $(\mathrm{E})$.

(I) Let $f(t)$ be a function of $C\left(1, t, t^{2}\right)$ on $(-\pi, \pi)$. Then

$$
\begin{array}{ll}
(-1)^{k+1} b_{k} \leqq(-1)^{k n+1} n b_{n k}, & k=1,2, \cdots, \\
& n=1,2, \cdots .
\end{array}
$$

Proof. Set $d \mu_{I}(t)=\left[(-1)^{k n+1} n \sin k n t-(-1)^{k+1} \sin k t\right] d t$. Simple integration yields

$$
I_{0} d \mu_{I}(t)=\frac{1}{k}\left[(-1)^{k n+1} \cos k n t-(-1)^{k+1} \cos k t\right],
$$

so that $I_{0} d \mu_{I}(\pi)=0$. Furthermore, $I_{0} d \mu_{I}(t)$ belongs to $C^{*}(1, t)$ by assertion (D). These facts imply that $d \mu_{I}(t)$ belongs to $\mathrm{C}^{*}\left(1, t, t^{2}\right)$. 
CoRollary I. If $f(t) \in C(1) \cap C\left(1, t, t^{2}\right)$, then we have

$$
\left|\frac{b_{n k}}{n}\right| \leqq\left|b_{k}\right| \leqq\left|n b_{n k}\right|, \quad \begin{aligned}
& k=1,2, \cdots, \\
& n=1,2, \cdots .
\end{aligned}
$$

(J) Let $f(t)$ be a function of $C\left(1, t, t^{2}, t^{3}\right)$ on $(-\pi, \pi)$. Then

$$
\begin{aligned}
(-1)^{k} a_{k} \leqq(-1)^{k n} n^{2} a_{n k}, \quad & k=1,2, \cdots, \\
& n=1,2, \cdots .
\end{aligned}
$$

Proof. Set $d \mu_{J}(t)=\left[(-1)^{k+1} \cos k t-(-1)^{k n+1} n^{2} \cos n k t\right] d t$. The familiar integration yields now

$$
I_{0} d \mu_{J}(t)=\frac{1}{k}\left[(-1)^{k n+1} n \sin k t-(-1)^{k+1} \sin k t\right],
$$

so that $I_{0} d \mu_{J}(\pi)=0$. Furthermore, $I_{0} d \mu_{J}(t)$ belongs to $C^{*}\left(1, t, t^{2}\right)$ by assertion 1). These facts imply that $d \mu_{J}(t)$ belongs to $C^{*}\left(1, t, t^{2}, t^{3}\right)$.

CoRollary J. If $f(t) \in C(1, t) \cap C\left(1, t, t^{2}, t^{3}\right)$, then we have

$$
\left|\frac{a_{n k}}{n^{2}}\right| \leqq\left|a_{k}\right| \leqq\left|n^{2} a_{n k}\right| . \quad \begin{aligned}
& k=1,2, \cdots, \\
& n=1,2, \cdots .
\end{aligned}
$$

Corollaries (I) and (J) imply the following theorem relating any two Euler-Fourier coefficients.

THEOREM 5. Let $P(n, m)$ denote the least common multiple of the natural numbers $m$ and $n$. The following inequalities are satisfied:

$$
\begin{aligned}
& \left|\frac{b_{m}}{P(m, n)}\right| \leqq\left|b_{n}\right| \leqq \mid \\
& \quad \text { for all } f(t) \in C(1) \cap C\left(1, t, t^{2}\right),
\end{aligned}
$$

and

$$
\begin{aligned}
\left|\frac{a_{m}}{P^{2}(m, n)}\right| \leqq\left|a_{n}\right| & \leqq\left|P^{2}(m, n) a_{m}\right|, \\
& \text { for all } f(t) \in C(1, t) \cap C\left(1, t, t^{2}, t^{3}\right) .
\end{aligned}
$$

(K) Let $f(t)$ be a convex function on $(-\pi, \pi)$. Then

$$
\int_{-\pi}^{\pi} t f(t) d t \leqq \frac{\pi^{3}}{3}\left(b_{1}-a_{1}\right)
$$

Proof. Consider the measure $d \mu_{k}(t)=\left(\sin t-\cos t-3 t / \pi^{2}\right) d t$. It 
is easily verified that both the first sign and the last sign of $d \mu_{K}(t)$ are $(+)$. A direct computation demonstrates that the "moment conditions" $\int_{-\pi}^{\pi} d \mu_{K}(t)=0$ and $\int_{-\pi}^{\pi} t d \mu_{K}(t)=0$ are satisfied. Moreover, an examination of the graph of $\sin t$-cost versus the graph of $3 t / \pi^{2}$ shows that $d \mu_{K}(t)$ has precisely two sign changes. Hence, Theorem B implies that $d \mu_{K}(t)$ belongs to $C^{*}(1, t)$; this is equivalent to assertion $(\mathrm{K})$.

(L) Let $f(t)$ be a monotone nondecreasing function on $(-\pi, \pi)$. Then

$$
\int_{-\pi}^{\pi} t^{2} f(t) d t \geqq \frac{2 \pi^{2}}{3}\left(\frac{a_{0}}{2}-b_{1}-a_{1}\right)
$$

Proof. Let $d \mu_{L}(t)=\left(\cos t+\sin t+3 t^{2} / 2 \pi^{2}-1 / 2\right) d t$. It is easily verified that $d \mu_{L}(t)=I_{0} d \mu_{K}(t) d t$. Since $I_{0} d \mu_{K}(\pi)=0$, we can conclude from assertion $(\mathrm{K})$ and the remark following equation (13) that $d \mu_{L}(t)$ belongs to $C^{*}(1)$, i.e., that (42) is indeed valid for all $f(t) \in C(1)$.

Since Theorems 1 and 2 specify necessary conditions for a measure to belong to a dual cone, some results of a negative nature are also to be expected. In fact, the following results can readily be deduced from Theorem 2.

THEOREM 6. Let $\left(u_{0}, \cdots, u_{2 n-1}\right), n \geqq 1$, be an ECT-system on $[-\pi, \pi]$. No finite linear inequality involving only $b_{i}^{\prime} s$ can be valid for all $f(t) \in C\left(u_{0}, \cdots, u_{2 n-1}\right)$.

Proof. It suffices to observe that a measure which is a linear combination of $\{\sin k t\}$ is an odd function on $(-\pi, \pi)$ and thus has an odd number of sign changes.

\section{A similar reasoning yields also}

THEOREM 7. Let $\left(u_{0}, \cdots, u_{2 n}\right), n \geqq 0$, be an ECT-system on $[-\pi, \pi]$. No finite linear inequality involving only $a_{i}^{\prime} s$ can be valid for all $f(t) \in C\left(u_{0}, \cdots, u_{2 n}\right)$.

One might conjecture, on the basis of assertion (D), that $\left\{\left|a_{n}\right|\right\}$ is a monotone decreasing sequence whenever $f(t)$ is a convex function. A computation of the corresponding $\left\{J_{i}\right\}$ and reliance on Theorem 1 , show, however, that neither $\left|a_{2}\right| \geqq\left|a_{3}\right|$ nor $\left|a_{3}\right| \geqq\left|a_{5}\right|$ are valid for all convex functions.

We conclude with the following 
REMARK. An inequality for the Euler-Fourier coefficients which holds for all functions of $C\left(1, t, \cdots, t^{n}\right)$ cannot hold, by Theorem 3 , for all functions of $C\left(1, t, \cdots, t^{m}\right), m \neq n$.

(b) Expansion in series of orthogonal polynomials. Let $\left\{P_{n}(t)\right\}_{n=0}^{\infty}$ be an orthonormal family of polynomials with respect to a weight function $w(t)$ on $(a, b)$, and let $P_{n}(t)$ be so normalized that the coefficient of $t^{n}$ is positive. Let $f(t)$ denote a function of $L_{2}(w(t) ; a, b)$ throughout this subsection, and let $c_{n}, n=0,1, \cdots$, denote the Fourier coefficients of $f(t)$ with respect to the system $\left\{P_{n}(t)\right\}$, i.e.,

$$
c_{n}=\int_{a}^{b} f(t) P_{n}(t) w(t) d t, \quad n=0,1, \cdots .
$$

Given that $f(t)$ belongs to a convexity cone, certain inequalities have to be satisfied by the coefficients $c_{n}, n=0,1, \ldots$. The derivation of such inequalities is the substance of this subsection.

THEOREM 8. Let $f(t)$ be a function of $C\left(1, t, \cdots, t^{n-1}\right)$. Then the following conditions are satisfied:

$$
c_{n} \geqq 0,
$$

and

$$
\frac{c_{n}}{P_{n}(b)} \geqq \frac{c_{n+1}}{P_{n+1}(b)}, \quad \frac{(-1)^{n+1} c_{n}}{P_{n}(a)} \leqq \frac{(-1)^{n+1} c_{n+1}}{P_{n+1}(a)}
$$

Proof. Set $d \mu_{1}(t)=P_{n}(t) w(t) d t$. Then relation (44) will follow if we show that $d \mu_{1}$ belongs to $C^{*}\left(1, t, \cdots, t^{n-1}\right)$. The orthogonality properties of the polynomials $P_{n}(t)$ imply that $d \mu_{1}$ satisfies the "moment conditions" (5). We recall now that $P_{n}(t)$ has $n$ simple zeros, i.e. $n$ sign changes, inside $(a, b)$ (see [10], Th. 3.3.1). Furthermore, since these are all the zeros, the normalization implies that the last sign of $d \mu_{1}$ on $(a, b)$ is $(+)$. Hence, relation (44) follows by appealing to Theorem B.

Consider next the measure

$$
d \mu_{2}(t)=\left[\frac{P_{n+1}(b)}{P_{n}(b)} P_{n}(t)-P_{n+1}(t)\right] w(t) d t .
$$

The "moment conditions" are clearly satisfied by $d \mu_{2}$ due to the orthogonality properties. Observe next that the polynomial

$$
P_{n+1}(b) P_{n}(t) / P_{n}(b)-P_{n+1}(t)
$$

has exactly $n$ sign changes inside $(a, b)$ (see [10], Th. 3.3.4). Since 
the $n+1$ - st zero is at $b$, the normalization implies that the polynomial must change its sign there from positive to negative. Hence, the last sign of $d \mu_{2}$ on $(a, b)$ is $(+)$ and the first of relations (45) is established by appealing to Theorem $B$.

Similarly, the measure

$$
d \mu_{3}(t)=\left[P_{n+1}(t)-\frac{P_{n+1}(a)}{P_{n}(a)} P_{n}(t)\right] w(t) d t
$$

has $n$ sign changes inside $(a, b)$ and an $n+1$-st sign change at $a$ (see [10], Th. 3.3.4). Its last sign on $(a, b)$ is $(+)$ and the "moment conditions" are satisfied. Thus, Theorem B implies that $d \mu_{3}$ belongs to $C^{*}\left(1, t, \cdots, t^{n-1}\right)$, i.e. that the inequality $c_{n+1} \geqq P_{n+1}(a) c_{n} / P_{n}(a)$ is valid for all $f(t) \in C\left(1, t, \cdots, t^{n-1}\right)$. Using the fact that

$$
(-1)^{n+1} P_{n+1}(a)>0,
$$

we obtain the second relation of (45).

COROLlaRY 8.1. If $f(t)$ is absolutely monotone on $(a, b)$ then $c_{n} \geqq 0, n=0,1, \cdots$, and the sequence $\left\{c_{n} / P_{n}(b)\right\}_{n=0}^{\infty}$ is monotone decreasing. It $f(t)$ is completely monotone on $(a, b)$ then

$$
(-1)^{n} c_{n} \geqq 0, n=0,1, \cdots,
$$

and the sequence $\left\{c_{n} / P_{n}(a)\right\}_{n=0}^{\infty}$ is monotone decreasing.

For special classes of orthogonal polynomials, some further results can be obtained. Let $(a, b)$ be a finite interval. Then, with no loss of generality we may assume that $a=-1, b=1$.

THEOREM 9. Let the weight function $w(t)$ be an even function and let $f(t)$ be a function of $C\left(t, t, \cdots, t^{n-1}\right)$. Then in addition to (44) and (45), we have

$$
\frac{c_{n}}{P_{n}(1)} \geqq \frac{c_{n+2}}{P_{n+2}(1)}
$$

Proof. Consider the measure

$$
d \mu(t)=\left[\frac{P_{n}(t)}{P_{n}(1)}-\frac{P_{n+2}(t)}{P_{n+2}(1)}\right] w(t) d t .
$$

The "moment conditions" (5) are satisfied by $d \mu$ by virtue of the orthogonality properties. Thus, by Theorem B, the polynomial

$$
Q(t) \equiv P_{n}(t) / P_{n}(1)-P_{n+2}(t) / P_{n+2}(1)
$$


has at least $n$ zeros inside $(-1,1)$. On the other hand, it can have at most $n$ zeros inside $(-1,1)$ since $Q(1)=0$ and the symmetry of $w(t)$ implies that $Q(-1)=0$. Hence, $Q(t)$ has exactly $n$ zeros inside $(-1,1)$. Noting that $t=1$ is the largest zero of $Q(t)$, we deduce from the normalization of the polynomials $P_{n}(t), n=0,1, \cdots$, that the last sign of $d \mu$ on $(-1,1)$ is $(+)$. Relation (46) follows now by appealing again to Theorem $\mathrm{B}$.

Note that the ultraspherical polynomials have a symmetric weight function, so that for them relations (44)-(46) are valid.

Consider now the expansion in terms of Tchebycheff polynomials. As a result of the strong affinity of these polynomials to the trigonometric functions, a general inequality for the coefficients of the expansion can be derived from the sole assumption that $f(x)$ is monotone nondecreasing.

Let $T_{n}(x), n=0,1, \cdots$, denote the $n$-th order Tchebycheff polynomial, and let the coefficients $a_{n}, n=0,1, \cdots$, be defined by

$$
a_{n}=\int_{-1}^{1} \frac{f(x) T_{n}(x)}{\sqrt{1-x^{2}}} d x, \quad n=0,1, \cdots
$$

THEOREM 10. Let $f(x)$ be a monotone nondecreasing function on $(-1,1)$. Then

$$
\left|a_{1}\right| \geqq\left|a_{n}\right|, \quad n=2,3, \cdots
$$

Proof. Note first that since $f(x) \in C(1)$, Theorem 8 implies that $a_{1} \geqq 0$. Hence, relation (48) is equivalent to $a_{1} \geqq\left|a_{n}\right|$.

We start by proving that $a_{1} \geqq a_{n}$. Consider the measure

$$
d \mu_{1}(x)=\frac{T_{1}(x)-T_{n}(x)}{\sqrt{1-x^{2}}} d x .
$$

We wish to prove that this measure belongs to $C^{*}(1)$ on $(-1,1)$. Making the monotone change of variable $x=\cos t, 0<t<\pi$, we see that our problem reduces to showing that

$$
d \mu_{2}(t)=(\cos n t-\cos t) d t
$$

belongs to $C^{*}(1)$ on $(0, \pi)$. The "moment condition $\int_{0}^{\pi} d \mu_{2}(t)=0$ is trivially satisfied. Furthermore, $d \mu_{2}(t)$ is negative on an interval extending to 0 and it is positive on an interval extending to $\pi$.

The elementary trigonometric identity

$$
\cos n t-\cos t=-2 \sin \frac{(n+1) t}{2} \sin \frac{(n-1) t}{2}
$$


shows that the zeros, i.e. the points of sign change, of $[\cos n t-\cos t]$ inside $(0, \pi)$ are the points $2 k \pi /(n+1), k=1,2, \cdots,[n / 2]$, and the points $2 k \pi /(n-1), k=1,2, \cdots,[(n-2) / 2]$. Thus, for $n=2$ or $n=3$, $d \mu_{2}$ changes sign only once so that the desired conclusion follows from Theorem B.

Assume now that $n \geqq 4$. Since $r /(n-1)<(r+2) /(n+1)$ for all $r, 1 \leqq r<n-1$, the ordered sequence of points of sign change of $d \mu_{2}$ inside $(0, \pi)$ is

$$
\frac{2 \pi}{n+1}, \frac{2 \pi}{n-1}, \frac{4 \pi}{n+1}, \cdots, \frac{2[(n-2) / 2] \pi}{n-1}, \frac{2[n / 2] \pi}{n+1} .
$$

The numbers $J_{i}, i=0,1, \cdots[(n-4) / 2]$, defined in (9), are thus given by

$$
\begin{aligned}
J_{i} & =\int_{2 i \pi /(n-1)}^{(2 i+2) \pi /(n-1)}(\cos n t-\cos t) d t \\
& =\frac{1}{n}\left[\sin \frac{(2 i+2) n \pi}{n-1}-\sin \frac{2 i n \pi}{n-1}\right]-\left[\sin \frac{(2 i+2) \pi}{n-1}-\sin \frac{2 i \pi}{n-1}\right] .
\end{aligned}
$$

Since $n \pi /(n-1)=\pi+\pi /(n-1)$, the expression for $J_{i}$ reduces to

$$
\begin{aligned}
J_{i}=\left(\frac{1}{n}-1\right)\left(\sin \frac{(2 i+2) \pi}{n-1}-\right. & \left.\sin \frac{2 i \pi}{n-1}\right), \\
& i=0,1, \cdots,[(n-4) / 2] .
\end{aligned}
$$

The last $J_{i}$ is given by

$$
\begin{aligned}
J_{[(n-2) / 2]} & =\int_{2[n-2) / 2] \pi /(n-1)}^{\pi}(\cos n t-\cos t) d t \\
& =-\frac{1}{n} \sin \frac{2[(n-2) / 2] \pi}{n-1}+\sin \frac{2[(n-2) / 2] \pi}{n-1} \\
& =\left(1-\frac{1}{n}\right) \sin \frac{2[(n-2) / 2]}{n-1}>0 .
\end{aligned}
$$

From (49) we can deduce that $J_{0}<0$ and that the sequence

$$
\left\{J_{i}, i=0,1, \cdots,[(n-2) / 2]\right\}
$$

has precisely one sign change, which is a change from negative to positive. Hence, by appealing to Lemma 2, we conclude that $d \mu_{2}(t)$ belongs to $C^{*}(1)$ on $(0, \pi)$. Thus $d \mu_{1}(x)$ belongs to $C^{*}(1)$ on $(-1,1)$ and the inequality $a_{1} \geqq a_{n}$ is established for all $f(x) \in C(1)$.

For the proof of the inequality $a_{1} \geqq-a_{n}$ we consider the measure $d \mu_{3}(x) \equiv\left[T_{1}(x)+T_{n}(x)\right]\left(1-x^{2}\right)^{-1 / 2} d x$ defined on $(-1,1)$. This measure belongs to $C^{*}(1)$ on $(-1,1)$ if, and only if $d \mu_{4}(t) \equiv-(\cos n t+\cos t) d t$ belongs to $C^{*}(1)$ on $(0, \pi)$. The proof that $d \mu_{4}(t)$ belongs to $C^{*}(1)$ 
proceeds in exactly the same way as the proof that $d \mu_{2}(t) \in C^{*}(1)$. We will not repeat the details. This completes the proof of the theorem.

The author wishes to express his deep gratitude to Professor Samuel Karlin for his guidance and inspiration. I also wish to thank the referee for his useful comments.

\section{REFERENCES}

1. R. E. Barlow and A. W. Marshall, Bounds for distributions with monotone hazard rate I, Ann. Math. Stat. 35 (1964), 1234-1257.

2. H. D. Brunk, Integral inequalities for functions with non decreasing increments, Pacific J. Math. 14 (1964), 783-793.

3. G. T. Cargo, Comparable means and generalized convexity, J. Math. Anal. Appl. 12 (1965), 387-392.

4. S. Karlin and A. Novikoff, Generalized convex inequalities, Pacific J. Math. 13 (1963), 1251-1279.

5. S. Karlin and W. Studden, Tchebycheff Systems with Applications in Analysis and Statistics, Interscience, New York, 1966.

6. S. Karlin and Z. Ziegler, Generalized absolutely monotone functions, Israel J. Math. 3 (1965), 173-180.

7. S. Karlin and Z. Ziegler, Chebyshevian spline functions, J. SIAM Num. Anal. 3 (1966), 514-543.

8. V. T. Levin and S. B. Steckin, Inequalities, Amer. Math. Soc. Translations, v. 14, 1-29.

9. G. Polya and G. Szego, Aufgaben und Lehrsatze aus der Analysis, Springer Verlag, Berlin, 1925.

10. G. Szego, Orthogonal polynomials, Amer. Math. Soc. Colloquium Pub. 23, New York, 1959.

11. Z. Ziegler, Generalized convexity cones, Pacific J. Math. 17 (1966), 561-580.

Received August 6, 1966, and in revised Form January 31, 1967. The main results of the first three sections of this paper were essentially contained in a $\mathrm{Ph}$. D. thesis submitted to Stanford University in 1964, while all the applications are new. This paper was presented at the International Congress of Mathematicians, Moscow, August 1966. 


\section{PACIFIC JOURNAL OF MATHEMATICS}

\section{EDITORS}

\author{
H. ROYDEN \\ Stanford University \\ Stanford, California
}

\author{
J. P. JANS \\ University of Washington \\ Seattle, Washington 98105
}

J. DugundJI

Department of Mathematics

Rice University

Houston, Texas 77001

RICHARD ARENS

University of California

Los Angeles, California 90024

\section{ASSOCIATE EDITORS}
E. F. BECKENBACH
B. H. NeumanN
F. WOLF
K. YOSIDA

\section{SUPPORTING INSTITUTIONS}

\author{
UNIVERSITY OF BRITISH COLUMBIA \\ CALIFORNIA INSTITUTE OF TECHNOLOGY \\ UNIVERSITY OF CALIFORNIA \\ MONTANA STATE UNIVERSITY \\ UNIVERSITY OF NEVADA \\ NEW MEXICO STATE UNIVERSITY \\ OREGON STATE UNIVERSITY \\ UNIVERSITY OF OREGON \\ OSAKA UNIVERSITY \\ UNIVERSITY OF SOUTHERN CALIFORNIA
}

\author{
STANFORD UNIVERSITY \\ UNIVERSITY OF TOKYO \\ UNIVERSITY OF UTAH \\ WASHINGTON STATE UNIVERSITY \\ UNIVERSITY OF WASHINGTON \\ AMERICAN MATHEMATICAL SOCIETY \\ CHEVRON RESEARCH CORPORATION \\ TRW SYSTEMS \\ NAVAL ORDNANCE TEST STATION
}

\footnotetext{
Mathematical papers intended for publication in the Pacific Journal of Mathematics should be in typed form or offset-reproduced, double spaced with large margins. Underline Greek letters in red, German in green, and script in blue. The first paragraph or two must be capable of being used separately as a synopsis of the entire paper. It should not contain references to the bibliography. Manuscripts may be sent to any one of the four editors. All other communications to the editors should be addressed to the managing editor, Richard Arens, University of California, Los Angeles, California 90024.

Each author of each article receives 50 reprints free of charge; additional copies may be obtained at cost in multiples of 50 .

The Pacific Journal of Mathematics is published monthly. Effective with Volume 16 the price per volume (3 numbers) is $\$ 8.00$; single issues, $\$ 3.00$. Special price for current issues to individual faculty members of supporting institutions and to individual members of the American Mathematical Society: $\$ 4.00$ per volume; single issues $\$ 1.50$. Back numbers are available.

Subscriptions, orders for back numbers, and changes of address should be sent to Pacific Journal of Mathematics, 103 Highland Boulevard, Berkeley 8, California.

Printed at Kokusai Bunken Insatsusha (International Academic Printing Co., Ltd.), 7-17, Fujimi 2-chome, Chiyoda-ku, Tokyo, Japan.

PUBLISHED BY PACIFIC JOURNAL OF MATHEMATICS, A NON-PROFIT CORPORATION

The Supporting Institutions listed above contribute to the cost of publication of this Journal, but they are not owners of publishers and have no responsibility for its content or policies.
} 


\section{Pacific Journal of Mathematics}

\section{Vol. 24, No. $3 \quad$ July, 1968}

Duane W. Bailey, On symmetry in certain group algebras ............ 413

Lawrence Peter Belluce and Surender Kumar Jain, Prime rings with a one-sided ideal satisfying a polynomial identity ................ 421

L. Carlitz, A note on certain biorthogonal polynomials ............. 425

Charles O. Christenson and Richard Paul Osborne, Pointlike subsets of a manifold ......................................... 431

Russell James Egbert, Products and quotients of probabilistic metric

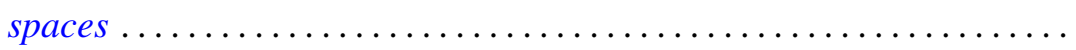

Moses Glasner, Richard Emanuel Katz and Mitsuru Nakai, Bisection into small annuli ..................................... 457

Karl Edwin Gustafson, A note on left multiplication of semigroup

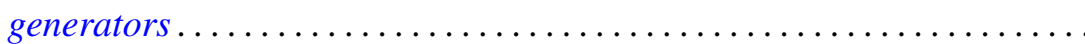

I. Martin (Irving) Isaacs and Donald Steven Passman, A characterization of groups in terms of the degrees of their characters. II ............. 467

Howard Wilson Lambert and Richard Benjamin Sher, Point-like

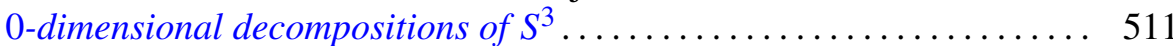

Oscar Tivis Nelson, Subdirect decompositions of lattices of width two ..... 519

Ralph Tyrrell Rockafellar, Integrals which are convex functionals . . . . . . . 525

James McLean Sloss, Reflection laws of systems of second order elliptic differential equations in two independent variables with constant coefficients ...

Bui An Ton, Nonlinear elliptic convolution equations of Wiener-Hopf type in a bounded region

Daniel Eliot Wulbert, Some complemented function spaces in $C(X)$

Zvi Ziegler, On the characterization of measures of the cone dual to a generalized convexity cone. 